\title{
Regularity Criteria on the 2D Anisotropic Magnetic Bénard Equations
}

\section{Dipendra Regmi*and Ramjee Sharma}

Department of Mathematics, University of North Georgia, 3820 Mundy Mill Rd, Oakwood, GA 30566, USA.

Received September 18, 2018; Accepted December 10, 2018

\begin{abstract}
In this paper, we study the global regularity issue of two dimensional incompressible magnetic Bénard equations with partial dissipation and magnetic diffusion. It remains open whether the smooth initial data produce solutions that are globally regular in time for all values of the parameters involved in the equations. We present conditional global regularity of the solutions. Moreover, we prove the global regularity for the slightly regularized system.
\end{abstract}

AMS subject classifications: 35Q35, 35B35, 35B65, 76D03

Key words: Global regularity, magnetic Bénard equations, partial dissipation

\section{Introduction}

This paper aims the global regularity of two dimensional magnetic Bénard equations. The standard two-dimensional incompressible magnetic Bénard equations can be written as

$$
\left\{\begin{array}{l}
u_{t}+u \cdot \nabla u=-\nabla p+v \Delta u+b \cdot \nabla b+\theta e_{2}, \\
b_{t}+u \cdot \nabla b=\eta \Delta b+b \cdot \nabla u, \\
\partial_{t} \theta+(u \cdot \nabla) \theta-\kappa \Delta \theta=u \cdot e_{2}, \\
\nabla \cdot u=0, \quad \nabla \cdot b=0, \\
u(x, y, 0)=u_{0}(x, y), b(x, y, 0)=b_{0}(x, y), \theta(x, y, 0)=\theta_{0}(x, y),
\end{array}\right.
$$

where $(x, y) \in \mathbb{R}^{2}, t \geq 0, u=\left(u_{1}(x, y, t), u_{2}(x, y, t)\right)$ denotes the $2 \mathrm{D}$ velocity field, $p=p(x, y, t)$ the pressure, $b=\left(b_{1}(x, y, t), b_{2}(x, y, t)\right)$ the magnetic field, $\theta(x, y, t)$ the temperature, $e_{2}=$ $(0,1)^{T}$ vertical unit vector, and $\nu, \eta$ and $\kappa$ are nonnegative real parameters.

\footnotetext{
*Corresponding author. Email addresses: dipendra.regmi@ung.edu (D. Regmi), ramjee.sharma@ung.edu
} (R. Sharma) 
A generalized 2D Magnetic Bénard equations can be written as

$$
\left\{\begin{array}{l}
u_{t}+u \cdot \nabla u=-\nabla p+v_{1} u_{x x}+v_{2} u_{y y}+b \cdot \nabla b+\theta e_{2} \\
b_{t}+u \cdot \nabla b=\eta_{1} b_{x x}+\eta_{2} b_{y y}+b \cdot \nabla u \\
\partial_{t} \theta+(u \cdot \nabla) \theta-\kappa_{1} \partial_{x x} \theta-\kappa_{2} \partial_{y y} \theta=u_{2} \\
\nabla \cdot u=0, \nabla \cdot b=0, \\
u(x, y, 0)=u_{0}(x, y), \quad b(x, y, 0)=b_{0}(x, y), \theta(x, y, 0)=\theta_{0}(x, y) .
\end{array}\right.
$$

If $v_{1}=v_{2}=v$ and $\eta_{1}=\eta_{2}=\eta$, and $\kappa_{1}=\kappa_{2}=\kappa$, then (1.2) reduces to the standard magnetic Bénard equations (1.1). This generalization is capable of modeling the motion of anisotropic fluids for which the diffusion properties in different directions are different.

In the absence of $\theta$, the magnetic Bénard equation reduces to magneto-hydrodynamic (MHD) equation. When all four parameters $v_{1}, v_{2}, \eta_{1}$, and $\eta_{2}$ are positive, the global regularity of the classical solution to 2D MHD equations has been established, see, e.g., [7], [19]. On the other hand, it remains a remarkable open problem whether classical solutions of the two-dimensional inviscid MHD equations, with all four parameters equal to zero, preserve their regularity for all time or have finite time blowup. Many attempts have been made but there are no any satisfactory results concerning the regularity of the solution. When $v_{1}>0, v_{2}=0, \eta_{1}=0$ and $\eta_{2}>0$ or when $v_{1}=0, v_{2}>0, \eta_{1}>0$ and $\eta_{2}=0$, the global regularity was established by $\mathrm{Cao}$ and $\mathrm{Wu}$ in [2]. Cao, Regmi, and Wu studied two dimensional MHD equations with horizontal dissipation and horizontal diffusion in [1]. They proved that any possible blow-up can be controlled by the $L^{\infty}$-norm of the horizontal components.

There are numerous papers related to two dimensional MHD equations $[1-8,16,20$, $23,25]$ and references therein, however only few papers are available related to magnetic Bénard equations. Y. Zhou et al. in [32] obtained the global regularity results related to the 2D magnetic Bénard problem with zero thermal conductivity. The authors used energy estimates as well as a well known property of Hardy space and Bounded Mean Value Oscillation (BMO) to prove the global regularity. Very recently, J. Cheng and L. Du in [6] proved the global well-posedness of the 2D Magnetic Bénard equations with mixed partial viscosity which included vertical or horizontal magnetic diffusion but no thermal diffusivity. The authors also obtained the global regularity as well as some conditional regularity of strong solutions of the problem with mixed partial viscosity, thus extending the existing result of the problem with the full dissipation. Likewise, the global regularity of generalized magnetic Bénard problem was studied by Y. Yamazaki in [28] by extending the existing results on Boussinesq equation and magneto-hydrodynamic equations. The author studied the problem with fractional Laplacian and logarithmic super criticality. The author showed that when the diffusive term has a full Laplacian, then a sufficiently smooth initial data evolves into a smooth solution under certain conditions. The author also presented additional global regularity criteria for the velocity field, magnetic field and the temperature field. 
This paper is devoted to the case when $v_{1}>0, v_{2}=0, \eta_{1}>0, \eta_{2}=0 . \kappa_{1}>0$, and $\kappa_{2}=0$, namely

$$
\left\{\begin{array}{l}
u_{t}+u \cdot \nabla u=-\nabla p+u_{x x}+b \cdot \nabla b+\theta e_{2}, \\
b_{t}+u \cdot \nabla b=b_{x x}+b \cdot \nabla u, \\
\partial_{t} \theta+(u \cdot \nabla) \theta-\partial_{x x} \theta=u_{2}, \\
\nabla \cdot u=0, \quad \nabla \cdot b=0, \\
u(x, y, 0)=u_{0}(x, y), b(x, y, 0)=b_{0}(x, y),
\end{array}\right.
$$

where we have set $v_{1}=\eta_{1}=\kappa_{1}=1$. This paper presents conditioned global regularity. More precisely, we prove the following theorem.

Theorem 1.1. Assume that $\left(u_{0}, b_{0}, \theta_{0}\right) \in H^{2}\left(\mathbb{R}^{2}\right), \nabla \cdot u_{0}=0$ and $\nabla \cdot b_{0}=0$. Then, (1.3) has $a$ unique classical solution $(u, b, \theta)$ satisfying, for any $T>0$,

$$
u, b, \theta, \partial_{x} u, \partial_{x} b, \partial_{x} \theta \in L^{\infty}\left([0, T] ; H^{2}\left(\mathbb{R}^{2}\right)\right)
$$

provided $\int_{0}^{T}\left\|u_{1}, b_{1}\right\|_{B M O}^{2} d t<\infty$.

This result is also true for the following system.

$$
\left\{\begin{array}{l}
u_{t}+u \cdot \nabla u=-\nabla p+u_{x x}+b \cdot \nabla b+\theta e_{2}, \\
b_{t}+u \cdot \nabla b=b_{x x}+b \cdot \nabla u, \\
\partial_{t} \theta+(u \cdot \nabla) \theta-\partial_{y y} \theta=u_{2}, \\
\nabla \cdot u=0, \nabla \cdot b=0, \\
u(x, y, 0)=u_{0}(x, y), b(x, y, 0)=b_{0}(x, y) .
\end{array}\right.
$$

If we follow the proof of Theorem 1.1, we can further prove the following theorem.

Theorem 1.2. Consider

$$
\left\{\begin{array}{l}
u_{t}+u \cdot \nabla u=-\nabla p+u_{y y}+b \cdot \nabla b+\theta e_{2}, \\
b_{t}+u \cdot \nabla b=b_{y y}+b \cdot \nabla u \\
\partial_{t} \theta+(u \cdot \nabla) \theta-\partial_{x x} \theta=u_{2} \\
\nabla \cdot u=0, \quad \nabla \cdot b=0, \\
u(x, y, 0)=u_{0}(x, y), \quad b(x, y, 0)=b_{0}(x, y) .
\end{array}\right.
$$

Assume that $\left(u_{0}, b_{0}, \theta_{0}\right) \in H^{2}\left(\mathbb{R}^{2}\right), \nabla \cdot u_{0}=0$ and $\nabla \cdot b_{0}=0$. Then, (1.3) has a unique classical solution $(u, b, \theta)$ satisfying, for any $T>0$,

$$
u, b, \theta, \partial_{x} u, \partial_{x} b, \partial_{x} \theta \in L^{\infty}\left([0, T] ; H^{2}\left(\mathbb{R}^{2}\right)\right)
$$

provided $\int_{0}^{T}\left\|u_{2}, b_{2}\right\|_{B M O}^{2} d t<\infty$.

Remark 1.1. (1) In [6], authors presented conditional regularity results for the system (1.3) and the results are different than we have presented here. 
(2) Our results improves the previously known results related to MHD equations with horizontal dissipation and magnetic diffusion in [1].

We furthermore consider slightly regularized version of (1.3), namely either for any $\epsilon, \delta>0$

$$
\left\{\begin{array}{l}
u_{t}+u \cdot \nabla u=-\nabla p+v_{2} u_{x x}+\epsilon(-\Delta)^{\delta} u+b \cdot \nabla b+\theta e_{2} \\
b_{t}+u \cdot \nabla b=\eta_{2} b_{x x}+\epsilon(-\Delta)^{\delta} b+b \cdot \nabla u \\
\partial_{t} \theta+(u \cdot \nabla) \theta-\partial_{x x} \theta=u_{2} \\
\nabla \cdot u=0, \quad \nabla \cdot b=0, \\
u(x, y, 0)=u_{0}(x, y), \quad b(x, y, 0)=b_{0}(x, y)
\end{array}\right.
$$

or

$$
\left\{\begin{array}{l}
u_{t}+u \cdot \nabla u=-\nabla p+v_{2} u_{x x}+\epsilon(-\Delta)^{\delta} u+b \cdot \nabla b+\theta e_{2} \\
b_{t}+u \cdot \nabla b=\eta_{2} b_{x x}+\epsilon(-\Delta)^{\delta} b+b \cdot \nabla u \\
\partial_{t} \theta+(u \cdot \nabla) \theta-\partial_{y y} \theta=u_{2} \\
\nabla \cdot u=0, \quad \nabla \cdot b=0, \\
u(x, y, 0)=u_{0}(x, y), \quad b(x, y, 0)=b_{0}(x, y)
\end{array}\right.
$$

We prove the following theorem.

Theorem 1.3. Assume that $\left(u_{0}, b_{0}, \theta_{0}\right) \in H^{2}\left(\mathbb{R}^{2}\right), \nabla \cdot u_{0}=0$ and $\nabla \cdot b_{0}=0$. Then, (1.6) or (1.7) has a unique classical solution $(u, b, \theta)$ satisfying, for any $T>0$,

$$
u, b, \theta, \partial_{x} u, \partial_{x} b, \partial_{x} \theta \in L^{\infty}\left([0, T] ; H^{2}\left(\mathbb{R}^{2}\right)\right) .
$$

Similar result is true if we consider the vertical dissipation and vertical magnetic diffusion.

The general approach to establish the global existence and regularity consists of two main steps. The first step is local existence and uniqueness and the second step is global a priori bounds. For this type of system, local existence follows from standard approach ( we omit here). We only concentrate to obtain the global bound. The main difficulty to obtain global bound for aforementioned system is $H^{1}$ bound. In $H^{1}$ bound, we encounter the terms $\int j \partial_{x} b_{1} \partial_{y} u_{1}$ and $\int j \partial_{x} u_{1} \partial_{y} b_{1}$. Unfortunately, we do not know how to bound the other two terms in order to close the inequality due to insufficient vertical dissipation and vertical magnetic diffusion. This is where the direct energy method breaks down and the global regularity problem becomes very hard.

The rest of this paper is divided into three sections. The last two sections are devoted to the proof for each of the theorems stated above.

\section{Preliminaries}

To simplify the notation, we will write $\|f\|_{2}$ for $\|f\|_{L^{2}}, \int f$ for $\int_{\mathbb{R}^{2}} f d x d y$ and write $\frac{\partial}{\partial x} f$, $\partial_{x} f$ or $f_{x}$ as the first partial derivative, and $\frac{\partial^{2}}{\partial x^{2}} f$ or $\partial_{x x} f$ as the second partial throughout the rest of this paper. BMO represents the bounded mean oscillation. 
The following anisotropic type Sobolev inequality will be frequently used. Its proof can be found in [2].

Lemma 2.1. If $f, g, h, \partial_{y} g, \partial_{x} h \in L^{2}\left(\mathbb{R}^{2}\right)$, then

$$
\iint_{\mathbb{R}^{2}}|f g h| d x d y \leq C\|f\|_{2}\|g\|_{2}^{\frac{1}{2}}\left\|\partial_{y} g\right\|_{2}^{\frac{1}{2}}\|h\|_{2}^{\frac{1}{2}}\left\|\partial_{x} h\right\|_{2}^{\frac{1}{2}},
$$

where $C$ is a constant.

The following simple fact on the boundedness of Riesz transforms will also be used.

Lemma 2.2. Let $f$ be divergence-free vector field such that $\nabla f \in L^{p}$ for $p \in(1, \infty)$. Then there exists a pure constant $C>0$ (independent of $p$ ) such that

$$
\|\nabla f\|_{L^{p}} \leq \frac{C p^{2}}{p-1}\|\nabla \times f\|_{L^{p}}
$$

\section{Proof of Theorem 1.1}

This section is devoted to the proof of Theorem 1.1. As explained in the introduction, it suffices to establish the global a priori bound for the solution in $H^{2}$. For the sake of clarity, we divide this process into two subsections. The first subsection proves the global $H^{1}$-bound while the second proves the global $H^{2}$-bound.

\section{1 $\quad H^{1}$-Bound}

We first state the global $L^{2}$-bound.

Lemma 3.1. Assume that $\left(u_{0}, b_{0}, \theta_{0}\right)$ satisfies the condition stated in Theorem 1.1. Let $(u, b, \theta)$ be the corresponding solution of (1.3). Then, $(u, b, \theta)$ obeys the following global $L^{2}$-bound,

$$
\left.\|u(t), b(t), \theta(t)\|_{L^{2}}^{2}+2 \int_{0}^{t}\left\|\partial_{x} u, \partial_{x} b, \partial_{x} \theta\right\|_{L^{2}}^{2}\right) d \tau \leq C\left(\left\|\left(u_{0}, b_{0}, \theta_{0}\right)\right\|_{2}^{2}\right)
$$

for any $t \geq 0$.

We can easily prove the global $L^{2}$ bound. Taking the $L^{2}$-inner product of $(u, b, \theta)$ with (1.3), respectively, and adding together yields

$$
\begin{aligned}
& \frac{1}{2} \frac{d}{d t}\left(\|u(t)\|_{L^{2}}^{2}+\|b(t)\|_{L^{2}}^{2}+\|\theta(t)\|_{L^{2}}^{2}\right)+\left\|\partial_{x} u(\tau)\right\|_{L^{2}}^{2}+\left\|\partial_{x} b(\tau)\right\|_{L^{2}}^{2}+\left\|\partial_{x} \theta(\tau)\right\|_{L^{2}}^{2} \\
= & \int \theta e_{2} \cdot u+\int u_{2} \theta \leq C\|u\|_{L^{2}}\|\theta\|_{L^{2}} .
\end{aligned}
$$


Combining together, we easily obtain the global $L^{2}$ bound.

$$
\|u(t), b(t), \theta(t)\|_{L^{2}}^{2}+2 \int_{0}^{t}\left\|\partial_{x} u(\tau)\right\|_{L^{2}}^{2} d \tau+2 \int_{0}^{t}\left\|\partial_{x} b(\tau)\right\|_{L^{2}}^{2} d \tau+2 \int\left\|\partial_{x} \theta\right\|_{2}^{2} d \tau \leq C,
$$

for any $0<t \leq T$, where $C$ depends only on the initial data. We next prove the global $H^{1}$-bound for $u, b$ and $\theta$. More precisely, we prove the following result.

Proposition 3.1. Assume that $\left(u_{0}, b_{0}, \theta_{0}\right)$ satisfies the condition stated in Theorem 1.1. Let $(u, b, \theta)$ be the corresponding solution of (1.3). Then $(u, b, \theta)$ satisfies, for any $T>0$,

$$
u, b, \theta \in C\left([0, T] ; H^{1}\left(\mathbb{R}^{2}\right)\right) .
$$

Consider the equation for $\omega=\nabla \times u$ and $j=\nabla \times b$ to estimate $H^{1}$,

$$
\left\{\begin{array}{l}
\omega_{t}+u \cdot \nabla \omega=\omega_{x x}+b \cdot \nabla j+\partial_{x} \theta \\
j_{t}+u \cdot \nabla j=j_{x x}+b \cdot \nabla \omega+2 \partial_{x} b_{1}\left(\partial_{x} u_{2}+\partial_{y} u_{1}\right)-2 \partial_{x} u_{1}\left(\partial_{x} b_{2}+\partial_{y} b_{1}\right) .
\end{array}\right.
$$

We then obtain

$$
\begin{aligned}
& \frac{1}{2} \frac{d}{d t}\left(\|\omega\|_{L^{2}}^{2}+\|j\|_{L^{2}}^{2}\right)+\left\|\omega_{x}\right\|_{L^{2}}^{2}+\left\|j_{x}\right\|_{L^{2}}^{2} \\
= & 2 \int j\left(\partial_{x} b_{1}\left(\partial_{x} u_{2}+\partial_{y} u_{1}\right)-2 \partial_{x} u_{1}\left(\partial_{x} b_{2}+\partial_{y} b_{1}\right)\right)+\int \partial_{x} \theta \omega \\
= & : I_{1}+I_{2}+I_{3}+I_{4}+I_{5} .
\end{aligned}
$$

For notational convenience, we will omit $d x d y$ from the spatial integral. The first term can be bounded by using Lemma 2.1

$$
I_{1}=\left|2 \int \partial_{x} b_{1} \partial_{x} u_{2} j\right| \leq C\left\|\partial_{x} u_{2}\right\|_{L^{2}}\left\|\partial_{x} b_{1}\right\|_{L^{2}}^{\frac{1}{2}}\left\|\partial_{x} \partial_{y} b_{1}\right\|_{L^{2}}^{\frac{1}{2}}\|j\|_{L^{2}}^{\frac{1}{2}}\left\|\partial_{x} j\right\|_{L^{2}}^{\frac{1}{2}}
$$

Applying Young's inequality and the simple fact that

$$
\left\|\partial_{x} b_{1}\right\|_{L^{2}} \leq\|j\|_{L^{2},} \quad\left\|\partial_{x} \partial_{y} b_{1}\right\|_{L^{2}} \leq\left\|\partial_{x} j\right\|_{L^{2}}
$$

we have

$$
I_{1} \leq \frac{1}{8}\left\|\partial_{x} j\right\|_{L^{2}}^{2}+C\left\|\partial_{x} u_{2}\right\|_{L^{2}}^{2}\|j\|_{L^{2}}^{2}
$$

Similarly,

$$
I_{3}=\left|2 \int \partial_{x} u_{1} \partial_{x} b_{2} j\right| \leq \frac{1}{8}\left\|\partial_{x} j\right\|_{L^{2}}^{2}+C\left\|\partial_{x} u_{1}\right\|_{L^{2}}^{2}\|j\|_{L^{2}}^{2}
$$

The terms $I_{2}$ and $I_{4}$ have to be handled differently. By integration by parts,

$$
I_{2}=2 \int \partial_{x} b_{1} \partial_{y} u_{1} j=-2 \int b_{1} \partial_{x} \partial_{y} u_{1} j-2 \int b_{1} \partial_{y} u_{1} \partial_{x} j
$$


which gives that

$$
I_{2} \leq \frac{1}{8}\left(\left\|\omega_{x}\right\|_{2}^{2}+\left\|j_{x}\right\|_{2}^{2}\right)+C\left\|b_{1}\right\|_{B M O}^{2}\left(\|j\|_{2}^{2}+\|\omega\|_{2}^{2}\right) .
$$

Similarly

$$
I_{4}=-2 \int \partial_{x} u_{1} \partial_{y} b_{1} j=-2 \int \partial_{x} u_{1} \partial_{y} b_{1} \partial_{x} b_{2}-2 \int \partial_{x} u_{1} \partial_{y} b_{1} \partial_{y} b_{1}=: I_{41}+I_{42}
$$

The last two terms admit

$$
\begin{aligned}
& I_{41} \leq \frac{1}{8}\left\|\omega_{x}\right\|_{2}^{2}+\frac{1}{8}\left\|j_{x}\right\|_{2}^{2}+C\|b\|_{2}^{2}\left\|\partial_{x} b\right\|_{2}^{2}\left(\|\omega\|_{2}^{2}+\|j\|_{2}^{2}\right), \\
& I_{42}=-4 \int u_{1} \partial_{y} b_{1} \partial_{x y} b_{1} \leq \frac{1}{8}\left\|\partial_{x} j\right\|_{2}^{2}+\frac{1}{8}\left\|u_{1}\right\|_{B M O}^{2}\|j\|_{2}^{2} .
\end{aligned}
$$

Furthermore,

$$
I_{5} \leq\left|\int \partial_{x} \theta \omega\right| \leq\|\theta\|_{2}^{2}+\left\|\omega_{x}\right\|_{2}^{2}
$$

After combining all inequalities

$$
\begin{array}{r}
\frac{1}{2} \frac{d}{d t}\left(\|\omega\|_{L^{2}}^{2}+\|j\|_{L^{2}}^{2}\right)+\left\|\partial_{x} \omega\right\|_{L^{2}}^{2}+\left\|\partial_{x} j\right\|_{L^{2}}^{2} \leq \frac{1}{2}\left\|\partial_{x} \omega\right\|_{2}^{2}+\frac{1}{2}\left\|\partial_{x} j\right\|_{2}^{2} \\
+C\left(\left\|u_{1}\right\|_{B M O}^{2}+\left\|b_{1}\right\|_{B M O}^{2}+\left\|\partial_{x} b\right\|_{2}^{2}+\|\theta\|_{2}^{2}\right)\left(\|j\|_{2}^{2}+\|\omega\|_{2}^{2}\right) .
\end{array}
$$

Gronwall's lemma yields,

$$
\|\omega\|_{L^{2}}^{2}+\|j\|_{L^{2}}^{2}+\int_{0}^{t}\left\|\partial_{x} \omega\right\|_{L^{2}}^{2}+\int_{0}^{t}\left\|\partial_{x} j\right\|_{L^{2}}^{2} \leq C e^{C \int_{0}^{t}\left(\left\|u_{1}\right\|_{B M O}^{2}+\left\|b_{1}\right\|_{B M O}^{2}+\left\|\partial_{x} b\right\|_{2}^{2}\right)} .
$$

Now we need to know the $H^{1}$ bound for $\theta$. Taking inner products of the third equation in (1.3) with $\Delta \theta$ and integrating by parts, we obtain

$$
\begin{gathered}
\frac{1}{2} \frac{d}{d t}\|\nabla \theta\|_{2}^{2}+\kappa\left\|\nabla \partial_{x} \theta\right\|_{2}^{2}=-\int \nabla u \cdot \nabla \theta \cdot \nabla \theta+(u \cdot \nabla) \nabla \theta \cdot \nabla \theta-\nabla u_{2} \cdot \nabla \theta \\
-\int \nabla u \cdot \nabla \theta \cdot \nabla \theta=-\int\left(\partial_{x} u_{1}\left(\partial_{x} \theta\right)^{2}+\partial_{x} u_{2} \partial_{x} \theta \partial_{y} \theta+\partial_{y} u_{1} \partial_{x} \theta \partial_{y} \theta+\partial_{y} u_{2}\left(\partial_{y} \theta\right)^{2}\right) \\
=J_{1}+J_{2}+J_{3}+J_{4} .
\end{gathered}
$$

By the divergence free condition $\int(u \cdot \nabla) \nabla \theta \cdot \nabla \theta=0$ and

$$
\int \nabla u_{2} \cdot \nabla \theta=\int \partial_{x} u_{2} \theta_{x}+\int \partial_{y} u_{2} \theta_{y}=: J_{5}+J_{6}
$$


we have

$$
\begin{aligned}
& J_{1}=-2 \int u_{2} \partial_{x} \theta \partial_{x y} \theta \leq C\left\|\partial_{x y} \theta\right\|_{2}^{\frac{3}{2}}\left\|u_{2}\right\|_{2}^{\frac{1}{2}}\left\|\partial_{x} \theta\right\|_{2}^{\frac{1}{2}} \leq \frac{1}{48}\left\|\nabla \partial_{x} \theta\right\|_{2}^{2}+C\left\|u_{2}\right\|_{2}^{2}\left\|\partial_{x} \theta\right\|_{2}^{2}, \\
& J_{2}=\int \partial_{x} u_{2} \partial_{x} \theta \partial_{y} \theta \leq C\left\|\partial_{x} u_{2}\right\|_{2}\left\|\partial_{x} \theta\right\|_{2}^{\frac{1}{2}}\left\|\partial_{y} \theta\right\|_{2}^{\frac{1}{2}}\left\|\partial_{x y} \theta\right\|_{2} \leq \frac{1}{48}\left\|\nabla \partial_{x} \theta\right\|_{2}^{2}+C\left\|\partial_{x} u_{2}\right\|_{2}^{2}\|\nabla \theta\|_{2}^{2} .
\end{aligned}
$$

Similarly,

$$
\begin{aligned}
J_{3}=\int \partial_{y} u_{1} \partial_{x} \theta \partial_{y} \theta \leq C\left\|\partial_{y} u_{1}\right\|_{2}\left\|\partial_{x} \theta\right\|_{2}^{\frac{1}{2}}\left\|\partial_{y} \theta\right\|_{2}^{\frac{1}{2}}\left\|\partial_{x y} \theta\right\|_{2} \\
\leq \frac{1}{48}\left\|\nabla \partial_{x} \theta\right\|_{2}^{2}+C\left\|\partial_{y} u\right\|_{2}^{2}\|\nabla \theta\|_{2}^{2}, \\
J_{4}=-2 \int u_{1} \partial_{x y} \theta \partial_{y} \theta \leq \frac{1}{48}\left\|\nabla \partial_{x} \theta\right\|_{2}^{2}+C\|u\|_{2}^{2}\|\nabla u\|_{2}^{2}\|\nabla \theta\|_{2}^{2}, \\
J_{5}=\int \partial_{x} u_{2} \theta_{x} \leq\left\|\partial_{x} u_{2}\right\|_{2}\left\|\partial_{x} \theta\right\|_{2}, \\
J_{6}=\int \partial_{y} u_{2} \theta_{y} \leq\left\|\partial_{y} u_{2}\right\|_{2}\left\|\partial_{y} \theta\right\|_{2} .
\end{aligned}
$$

Combining all inequalities together with Gronwall's lemma yields,

$$
\|\nabla \theta(t)\|_{2}^{2}+\int_{0}^{t}\left\|\nabla \partial_{x} \theta\right\|_{2}^{2} \leq C
$$

for any $t \leq T$. This completes the global $H^{1}$ bound for $(u, b, \theta)$.

\section{2 $H^{2}$-bound}

Taking the inner with of (3.2) with $(\Delta \omega, \Delta j)$ yields

$$
\begin{aligned}
& \frac{1}{2} \frac{d}{d t}\|\nabla \omega\|_{2}^{2}+\left\|\nabla \omega_{x}\right\|_{2}^{2}=-\int \nabla \omega \cdot \nabla u \cdot \nabla \omega d x d y+\int \nabla \omega \cdot \nabla b \cdot \nabla j d x d y \\
& \quad+\int b \cdot \nabla(\nabla j) \cdot \nabla \omega d x d y+\int \partial_{x} \theta \Delta \omega d x d y, \\
& \frac{1}{2} \frac{d}{d t}\|\nabla j\|_{2}^{2}+\left\|\nabla j_{x}\right\|_{2}^{2}=-\int \nabla j \cdot \nabla u \cdot \nabla j d x d y+\int \nabla j \cdot \nabla b \cdot \nabla \omega d x d y \int b \cdot \nabla(\nabla \omega) \cdot \nabla j d x d y \\
& \quad+2 \int \nabla\left[\partial_{x} b_{1}\left(\partial_{x} u_{2}+\partial_{y} u_{1}\right)\right] \cdot \nabla j d x d y-2 \int \nabla\left[\partial_{x} u_{1}\left(\partial_{x} b_{2}+\partial_{y} b_{1}\right)\right] \cdot \nabla j d x d y .
\end{aligned}
$$

Adding above equations and integrating by parts, we obtain

$$
\frac{1}{2} \frac{d}{d t}\left(\|\nabla \omega\|_{2}^{2}+\|\nabla j\|_{2}^{2}+\|\Delta \theta\|_{2}^{2}\right)+\left\|\nabla \omega_{x}\right\|_{2}^{2}+\left\|\nabla j_{x}\right\|_{2}^{2}+\|\nabla \Delta \theta\|_{2}^{2}=: \sum_{i=1}^{8} K_{i},
$$


where

$$
\begin{aligned}
& K_{1}=-\int \nabla \omega \cdot \nabla u \cdot \nabla \omega d x d y, \quad K_{2}=-\int \nabla j \cdot \nabla u \cdot \nabla j d x d y, \\
& K_{3}=2 \int \nabla \omega \cdot \nabla b \cdot \nabla j d x d y, \quad K_{4}=2 \int \nabla\left[\partial_{x} b_{1}\left(\partial_{x} u_{2}+\partial_{y} u_{1}\right)\right] \cdot \nabla j d x d y, \\
& K_{5}=-2 \int \nabla\left[\partial_{x} u_{1}\left(\partial_{x} b_{2}+\partial_{y} b_{1}\right)\right] \cdot \nabla j d x d y, \quad K_{6}=\int \partial_{x} \theta \Delta \omega d x d y, \\
& K_{7}=\int \Delta(u \cdot \nabla) \theta \Delta \theta d x d y, \quad K_{8}=\int \Delta u_{2} \Delta \theta d x d y .
\end{aligned}
$$

Observe that

$$
\begin{aligned}
K_{1} & =\int(\nabla \omega \cdot \nabla u \cdot \nabla \omega) d x d y \\
& =\int\left(\partial_{x} u_{1} \omega_{x}^{2}+\partial_{x} u_{2} \omega_{x} \omega_{y}+\partial_{y} u_{1} \omega_{x} \omega_{y}+\partial_{y} u_{2} \omega_{y}^{2}\right) d x d y \\
& =: K_{11}+K_{12}+K_{13}+K_{14} .
\end{aligned}
$$

By Lemma 2.1, we have

$$
K_{11} \leq C\left\|\partial_{x} u_{1}\right\|_{2}\left\|\omega_{x}\right\|_{2}^{\frac{1}{2}}\left\|\omega_{x x}\right\|_{2}^{\frac{1}{2}}\left\|\omega_{x}\right\|_{2}^{\frac{1}{2}}\left\|\omega_{x y}\right\|_{2}^{\frac{1}{2}} \leq \frac{1}{48}\left\|\nabla \omega_{x}\right\|_{2}^{2}+C\|\omega\|_{2}^{2}\|\nabla \omega\|_{2}^{2} .
$$

Similarly, we obtain

$$
\begin{aligned}
K_{12} & \leq C\left\|\partial_{x} u_{2}\right\|{ }_{2}\left\|\omega_{x}\right\|_{2}^{\frac{1}{2}}\left\|\omega_{x y}\right\|_{2}^{\frac{1}{2}}\left\|\omega_{y}\right\|_{2}^{\frac{1}{2}}\left\|\omega_{x y}\right\|_{2}^{\frac{1}{2}} \\
& \leq C\|\omega\|_{2}\|\nabla \omega\|_{2}\left\|\nabla \omega_{x}\right\|_{2} \leq \frac{1}{48}\left\|\nabla \omega_{x}\right\|_{2}^{2}+\|\omega\|_{2}^{2}\|\nabla \omega\|_{2}^{2}
\end{aligned}
$$

Furthermore,

$$
K_{13} \leq \frac{1}{48}\left\|\nabla \omega_{x}\right\|_{2}^{2}+\|\omega\|_{2}^{2}\|\nabla \omega\|_{2}^{2}, \quad K_{14} \leq \frac{1}{48}\left\|\nabla \omega_{x}\right\|_{2}^{2}+C\|\omega\|_{2}^{\frac{2}{3}}\left\|\omega_{x}\right\|_{2}^{\frac{2}{3}}\|\nabla \omega\|_{2}^{2} .
$$

Similarly,

$$
\begin{aligned}
K_{2} & =-\int \nabla j \cdot \nabla u \cdot \nabla j d x d y \\
& =\int \partial_{x} u_{1} j_{x}^{2}+\partial_{y} u_{1} j_{x} j_{y}+\partial_{y} u_{2} j_{y}^{2}+\partial_{x} u_{2} j_{x} j_{y}=: K_{21}+K_{22}+K_{23}+K_{24} .
\end{aligned}
$$

Observe that

$$
\begin{aligned}
K_{21} & \leq C\left\|j_{x}\right\|_{2}\left\|\partial_{x} u_{1}\right\|_{2}^{\frac{1}{2}}\left\|\partial_{x y} u_{1}\right\|_{2}^{\frac{1}{2}}\left\|j_{x}\right\|_{2}^{\frac{1}{2}}\left\|j_{x x}\right\|_{2}^{\frac{1}{2}} \\
& \leq C\|\omega\|_{2}^{\frac{1}{2}}\left\|\omega_{x}\right\|_{2}^{\frac{1}{2}}\|\nabla j\|^{\frac{3}{2}}\left\|\nabla j_{x}\right\|_{2}^{\frac{1}{2}} \leq \frac{1}{48}\left\|\nabla j_{x}\right\|_{2}^{2}+C\|\omega\|_{2}^{\frac{2}{3}}\left\|\omega_{x}\right\|_{2}^{\frac{2}{3}}\|\nabla j\|_{2}^{2}, \\
K_{22} & \leq \frac{1}{48}\left\|\nabla j_{x}\right\|_{2}^{2}+C\|\omega\|_{2}^{\frac{2}{3}}\left\|\omega_{x}\right\|_{2}^{\frac{2}{3}}\|\nabla j\|_{2}^{2}, \quad K_{23} \leq \frac{1}{48}\left\|\nabla j_{x}\right\|_{2}^{2}+\|\omega\|_{2}^{\frac{2}{3}}\left\|\omega_{x}\right\|_{2}^{\frac{2}{3}}\|\nabla j\|_{2}^{2}, \\
K_{24} & \leq \frac{1}{48}\left\|\nabla j_{x}\right\|_{2}^{2}+C\|\omega\|_{2}^{2}\|\nabla j\|_{2}^{2} .
\end{aligned}
$$


On the other hand,

$$
K_{3}=\int \omega_{x} \partial_{x} b_{1} j_{x}+\omega_{x} \partial_{x} b_{2} j_{y}+\omega_{y} \partial_{y} b_{1} j_{x}+\omega_{y} \partial_{y} b_{2} j_{y}=: K_{31}+K_{32}+K_{33}+K_{34} .
$$

Observe that

$$
\begin{aligned}
K_{31} & =\int \omega_{x} \partial_{x} b_{1} j_{x} \leq C\left\|\partial_{x} b_{1}\right\|_{2}\left\|\omega_{x}\right\|_{2}^{\frac{1}{2}}\left\|\omega_{x y}\right\|_{2}^{\frac{1}{2}}\left\|j_{x}\right\|_{2}^{\frac{1}{2}}\left\|j_{x x}\right\|_{2}^{\frac{1}{2}} \\
& \leq C\|j\|_{2}\|\nabla \omega\|_{2}^{\frac{1}{2}}\left\|\nabla \omega_{x}\right\|_{2}^{\frac{1}{2}}\|\nabla j\|_{2}^{\frac{1}{2}}\left\|\nabla j_{x}\right\|_{2}^{\frac{1}{2}} \\
& \leq \frac{1}{48}\left\|\nabla \omega_{x}\right\|_{2}^{2}+\frac{1}{48}\left\|\nabla j_{x}\right\|_{2}^{2}+C\|j\|_{2}^{2}\left(\|\nabla \omega\|_{2}^{2}+\|\nabla j\|_{2}^{2}\right) .
\end{aligned}
$$

Similarly,

$$
\begin{aligned}
& K_{32} \leq \frac{1}{48}\left\|\nabla \omega_{x}\right\|_{2}^{2}+\frac{1}{48}\left\|\nabla j_{x}\right\|_{2}^{2}+C\|j\|_{2}^{2}\left(\|\nabla \omega\|_{2}^{2}+\|\nabla j\|_{2}^{2}\right), \\
& K_{33} \leq \frac{1}{48}\left(\left\|\nabla w_{x}\right\|_{2}^{2}+\left\|\nabla j_{x}\right\|_{2}^{2}\right)+C\|j\|_{2}^{2}\left(\|\nabla \omega\|_{2}^{2}+\|\nabla j\|_{2}^{2}\right), \\
& K_{34} \leq \frac{1}{48}\left\|\nabla j_{x}\right\|_{2}^{2}+C\left\|j_{x}\right\|_{2}^{2}\|\nabla j\|_{2}^{2}+C\left\|_{j}\right\|_{2}\|\nabla \omega\|_{2}^{2} .
\end{aligned}
$$

Note that

$$
\begin{aligned}
K_{4} & =2 \int \nabla\left[\partial_{x} b_{1}\left(\partial_{x} u_{2}+\partial_{y} u_{1}\right)\right] \cdot \nabla j d x d y \\
& =2 \int \partial_{x}\left[\partial_{x} b_{1}\left(\partial_{x} u_{2}+\partial_{y} u_{1}\right)\right] j_{x}+\partial_{y}\left[\partial_{x} b_{1}\left(\partial_{x} u_{2}+\partial_{y} u_{1}\right)\right] j_{y} d x d y \\
& =: K_{41}+K_{42} .
\end{aligned}
$$

Observe that

$$
\begin{aligned}
& K_{41}=-2 \int \partial_{x} b_{1}\left(\partial_{x} u_{2}+\partial_{y} u_{1}\right) j_{x x} \\
\leq & C\left(\left\|\partial_{x} b_{1}\right\|_{2}^{\frac{1}{2}}\left\|\partial_{x y} b_{1}\right\|_{2}^{\frac{1}{2}}\left\|\partial_{x} u_{2}\right\|_{2}^{\frac{1}{2}}\left\|\partial_{x y} u_{2}\right\|_{2}^{\frac{1}{2}}+C\left\|\partial_{x} b_{1}\right\|_{2}^{\frac{1}{2}}\left\|\partial_{x y} b_{1}\right\|_{2}^{\frac{1}{2}}\left\|\partial_{y} u_{1}\right\|_{2}^{\frac{1}{2}}\left\|\partial_{x y} u_{1}\right\|_{2}^{\frac{1}{2}}\right)\left\|j_{x x}\right\|_{2} \\
\leq & C\|j\|_{2}^{\frac{1}{2}}\|\nabla j\|_{2}^{\frac{1}{2}}\|\omega\|_{2}^{\frac{1}{2}}\left\|\omega_{y}\right\|_{2}^{\frac{1}{2}}\left\|\nabla j_{x}\right\|_{2} \\
\leq & \frac{1}{48}\left\|\nabla j_{x}\right\|_{2}^{2}+C\|\omega\|_{2}\|j\|_{2}\left(\|\nabla \omega\|_{2}^{2}+\|\nabla j\|_{2}^{2}\right) .
\end{aligned}
$$

We further split $K_{42}$ into four parts

$$
\begin{aligned}
K_{42} & =2 \int\left(\partial_{x y} b_{1} \partial_{x} u_{2}+\partial_{x} b_{1} \partial_{x y} u_{2}+\partial_{x y} b_{1} \partial_{y} u_{1}+\partial_{x} b_{1} \partial_{y y} u_{1}\right) j_{y} d x d y \\
& =: K_{421}+K_{422}+K_{423}+K_{424}
\end{aligned}
$$


We also provide estimates to each of the four terms:

$$
\begin{aligned}
K_{421} & \leq C\left\|\partial_{x y} b_{1}\right\|_{2}\left\|\partial_{x} u_{2}\right\|_{2}^{\frac{1}{2}}\left\|\partial_{x y} u_{2}\right\|_{2}^{\frac{1}{2}}\left\|j_{y}\right\|_{2}^{\frac{1}{2}}\left\|j_{x y}\right\|_{2}^{\frac{1}{2}} \\
& \leq C\left\|j_{x}\right\|_{2}\|\omega\|_{2}^{\frac{1}{2}}\left\|\omega_{x}\right\|_{2}^{\frac{1}{2}}\|\nabla j\|_{2}^{\frac{1}{2}}\left\|\nabla j_{x}\right\|_{2}^{\frac{1}{2}} \\
& \leq C\|\nabla j\|_{2}^{\frac{3}{2}}\left\|_{2}\right\| \omega\left\|_{2}^{\frac{1}{2}}\right\| \nabla j_{x}\left\|_{2}^{\frac{1}{2}}\right\| \omega_{x} \|_{2}^{\frac{1}{2}} \\
& \leq \frac{1}{48}\left\|\nabla j_{x}\right\|_{2}^{2}+\|\omega\|_{2}^{\frac{2}{3}}\left\|\omega_{x}\right\|_{2}^{\frac{2}{3}}\|\nabla j\|_{2}^{2} .
\end{aligned}
$$

Similarly

$$
\begin{aligned}
& K_{422} \leq \frac{1}{48}\left\|\nabla j_{x}\right\|_{2}^{2}+C\left\|j_{x}\right\|_{2}^{2}\|\nabla j\|_{2}^{2}+C\|j\|_{2}\|\nabla \omega\|_{2}^{2}, \\
& K_{423} \leq \frac{1}{48}\left\|\nabla j_{x}\right\|_{2}^{2}+C\|\omega\|_{2}\|\nabla j\|_{2}^{2}+C\left\|j_{x}\right\|_{2}^{2}\|\nabla \omega\|_{2}^{2}, \\
& K_{424} \leq \frac{1}{48}\left\|\nabla \omega_{x}\right\|_{2}^{2}+C\left\|j_{x}\right\|_{2}^{2}\|\nabla \omega\|_{2}^{2}+C\|j\|_{2}\|\nabla j\|_{2}^{2} .
\end{aligned}
$$

Two more terms remain to be estimated. First, we work on $K_{5}$ :

$$
\begin{aligned}
K_{5} & =-2 \int \nabla\left[\partial_{x} u_{1}\left(\partial_{x} b_{2}+\partial_{y} b_{1}\right)\right] \cdot \nabla j d x d y \\
& =-2 \int \partial_{x}\left[\partial_{x} u_{1}\left(\partial_{x} b_{2}+\partial_{y} b_{1}\right)\right] j_{x}+\partial_{y}\left[\left(\partial_{x} u_{1}\left(\partial_{x} b_{2}+\partial_{y} b_{1}\right)\right] j_{y} d x d y\right. \\
& =: K_{51}+K_{52} .
\end{aligned}
$$

\section{Observe that}

$$
\begin{aligned}
K_{51} \leq & C\left\|\partial_{x} u_{1}\right\|_{2}^{\frac{1}{2}}\left\|\partial_{x y} u_{1}\right\|_{2}^{\frac{1}{2}}\left\|\partial_{x} b_{2}\right\|_{2}^{\frac{1}{2}}\left\|\partial_{x x} b_{2}\right\|_{2}^{\frac{1}{2}}\left\|j_{x x}\right\|_{2} \\
& +C\left\|\partial_{x} u_{1}\right\|_{2}^{\frac{1}{2}}\left\|\partial_{x y} u_{1}\right\|_{2}^{\frac{1}{2}}\left\|\partial_{y} b_{1}\right\|_{2}^{\frac{1}{2}}\left\|\partial_{x y} b_{1}\right\|_{2}^{\frac{1}{2}}\left\|j_{x x}\right\|_{2}^{\frac{1}{2}} \\
\leq & C\|\omega\|_{2}^{\frac{1}{2}}\|\nabla \omega\|_{2}^{\frac{1}{2}}\|j\|_{2}^{\frac{1}{2}}\|\nabla j\|_{2}^{\frac{1}{2}}\left\|\nabla j_{x}\right\|_{2} \\
\leq & \frac{1}{48}\left\|\nabla j_{x}\right\|_{2}^{2}+C\|\omega\|_{2}\|j\|_{2}\left(\|\nabla \omega\|_{2}^{2}+\|\nabla j\|_{2}^{2}\right), \\
K_{52}= & -2 \int\left(\partial_{x y} u_{1} \partial_{x} b_{2}+\partial_{x} u_{1} \partial_{x y} b_{2}+\partial_{x y} u_{1} \partial_{y} b_{1}+\partial_{x} u_{1} \partial_{y y} b_{1}\right) j_{y} d x d y \\
= & : K_{521}+K_{522}+K_{523}+K_{524} .
\end{aligned}
$$


Note that $K_{521}$ and $K_{522}$ can be bounded as $K_{422}$ and $K_{421}$. We only need to bound $K_{523}$ and $K_{524}$.

$$
\begin{aligned}
K_{523} & =-2 \int \partial_{x y} u_{1} \partial_{y} b_{1} j_{y} d x d y \\
& \leq C\left\|\partial_{x y} u_{1}\right\|_{2}^{\frac{1}{2}}\left\|\partial_{x y y} u_{1}\right\|_{2}^{\frac{1}{2}}\left\|\partial_{y} b_{1}\right\|_{2}^{\frac{1}{2}}\left\|\partial_{x y} b_{1}\right\|_{2}^{\frac{1}{2}}\left\|j_{y}\right\|_{2} \\
& \leq C\left\|\nabla \omega_{x}\right\|_{2}^{\frac{1}{2}}\left\|j_{x}\right\|_{2}^{\frac{1}{2}}\|\nabla \omega\|_{2}^{\frac{1}{2}}\|j\|_{2}^{\frac{1}{2}}\|\nabla j\|_{2} \\
& \leq \frac{1}{48}\left\|\nabla \omega_{x}\right\|_{2}^{2}+\left\|j_{x}\right\|_{2}^{2}\|\nabla \omega\|_{2}^{2}+\|j\|_{2}\|\nabla j\|_{2}^{2} .
\end{aligned}
$$

Finally, note that

$$
K_{6} \leq\left\|\nabla \partial_{x} \theta\right\|_{2}^{2}\|\nabla \omega\|_{2} \leq \frac{1}{48}\left\|\nabla \partial_{x} \theta\right\|_{2}^{2}+C\|\nabla \omega\|_{2}^{2} .
$$

After combining all inequalities, together with the Gronwall's lemma yields

$$
\|\nabla \omega\|_{2}^{2}+\|\nabla j\|_{2}^{2}+\int_{0}^{t}\left(\left\|\nabla \partial_{x} \omega(\tau)\right\|_{2}^{2}+\left\|\nabla \partial_{x} j(\tau)\right\|_{2}^{2}\right) d \tau \leq C .
$$

For the global bound for $\|\Delta \theta\|_{2}$, applying $\Delta$ to the third equation in (1.3) with $\Delta \theta$ and integrating by parts, we obtain

$$
\frac{1}{2} \frac{d}{d t}\|\Delta \theta\|_{2}^{2}+\left\|\Delta \partial_{x} \theta\right\|_{2}^{2}=\int \Delta(u \cdot \nabla) \theta \Delta \theta d x d y+\int \Delta u_{2} \Delta \theta d x d y .
$$

By Lemma 2.1

$$
\int \Delta(u \cdot \nabla) \theta \Delta \theta d x d y=\int \Delta u_{1} \theta_{x} \Delta \theta+2 \nabla u_{1} \cdot \nabla \theta_{x} \Delta \theta+\Delta u_{2} \theta_{y} \Delta \theta+2 \nabla u_{2} \cdot \nabla \theta_{y} \Delta \theta .
$$

Now we observe that

$$
\begin{aligned}
\left|\int \Delta u_{1} \theta_{x} \Delta \theta\right| & \leq\left\|\Delta u_{1}\right\|_{2}\left\|\theta_{x}\right\|_{2}^{\frac{1}{2}}\left\|\theta_{x y}\right\|_{2}^{\frac{1}{2}}\|\Delta \theta\|_{2}^{\frac{1}{2}}\left\|\Delta \theta_{x}\right\|_{2}^{\frac{1}{2}} \\
& \leq \frac{1}{48}\left\|\Delta \theta_{x}\right\|_{2}^{2}+C\left\|\theta_{x}\right\|_{2}^{\frac{2}{3}}\left\|\theta_{x y}\right\|_{2}^{\frac{2}{3}}\left\|\Delta u_{1}\right\|_{2}^{\frac{4}{3}}\|\Delta \theta\|_{2}^{\frac{2}{3}} \\
& \leq \frac{1}{48}\left\|\Delta \theta_{x}\right\|_{2}^{2}+C\left\|\theta_{x}\right\|_{2}^{\frac{2}{3}}\left\|\theta_{x y}\right\|_{2}^{\frac{2}{3}}\left(\left\|\Delta u_{1}\right\|_{2}^{2}+\|\Delta \theta\|_{2}^{2}\right) .
\end{aligned}
$$

Similarly

$$
\begin{aligned}
& \left|\int 2 \nabla u_{1} \cdot \nabla \theta_{x} \Delta \theta\right| \leq \frac{1}{48}\left\|\Delta \theta_{x}\right\|_{2}^{2}+C\left\|\nabla u_{1}\right\|_{2}^{\frac{2}{3}}\left\|\nabla \partial_{x} u_{1}\right\|_{2}^{\frac{2}{3}}\|\Delta \theta\|_{2}^{2}, \\
& \left|\int \Delta u_{2} \theta_{y} \Delta \theta\right| \leq \frac{1}{48}\left\|\Delta \partial_{x} u_{2}\right\|_{2}^{2}+\frac{1}{48}\left\|\Delta \theta_{x}\right\|_{2}^{2}+C\left\|\theta_{y}\right\|_{2}^{2}\left(\left\|\Delta u_{2}\right\|_{2}^{2}+\|\Delta \theta\|_{2}^{2}\right), \\
& \left|\int 2 \nabla u_{2} \cdot \nabla \theta_{y} \Delta \theta\right| \leq \frac{1}{48}\left\|\Delta \theta_{x}\right\|_{2}^{2}+C\left\|\nabla u_{2}\right\|_{2}^{\frac{2}{3}}\left\|\nabla \partial_{x} u_{1}\right\|_{2}^{\frac{2}{3}}\|\Delta \theta\|_{2}^{2} .
\end{aligned}
$$


Finally,

$$
\left|\int \Delta u_{2} \Delta \theta d x d y\right| \leq \frac{1}{48}\left\|\Delta u_{2}\right\|_{2}^{2}+C\|\Delta \theta\|_{2}^{2}
$$

Collecting all inequalities and applying Gronwall's lemma, we obtain

$$
\|\Delta \theta\|_{2}^{2}+\int_{0}^{t}\left\|\Delta \partial_{x} \theta\right\|_{2}^{2} \leq C
$$

for any $t \leq T$. This completes the proof of Theorem 1.1.

\section{Global regularity of slightly regularized system}

This section establishes that (1.6) possesses global regular solutions if the initial data are sufficiently smooth. More precisely, we prove Theorem 1.3. The difficult part to show the global regularity is obtaining global $H^{1}$ bound since global $H^{2}$-bound is similar to Theorem 1.1.

To obtain the global bound for the $H^{1}$-norm, we take advantage of the vorticity formulation. Taking the curl of (1.6), we find that $\omega=\nabla \times u$ and $j=\nabla \times b$ satisfy

$$
\left\{\begin{array}{l}
\omega_{t}+u \cdot \nabla \omega+\epsilon(-\Delta)^{\delta} \omega=b \cdot \nabla j+\omega_{x x}+\partial_{x} \theta \\
j_{t}+u \cdot \nabla j+\epsilon(-\Delta)^{\delta} j=b \cdot \nabla \omega+j_{x x}+2 \partial_{x} b_{1}\left(\partial_{y} u_{1}+\partial_{x} u_{2}\right)-2 \partial_{x} u_{1}\left(\partial_{y} b_{1}+\partial_{x} b_{2}\right) .
\end{array}\right.
$$

The main difficulty to show the global regularity is $H^{1}$-bound for $(u, b)$. We only sketch the proof of $H^{1}$ bound.

Taking the inner product of $(4.1)$ with $(\omega, j)$ and integrating by parts, we obtain

$$
\begin{aligned}
& \frac{1}{2} \frac{d}{d t}\left(\|\omega\|_{2}^{2}+\|j\|_{2}^{2}\right)+\left\|\partial_{x} \omega\right\|_{2}^{2}+\left\|\partial_{x} j\right\|_{2}^{2}+\epsilon\left\|\Lambda^{\delta} \omega\right\|_{2}^{2}+\epsilon\left\|\Lambda^{\delta} j\right\|_{2}^{2} \\
= & : J_{1}+J_{2}+J_{3}+J_{4}+J_{5},
\end{aligned}
$$

where

$$
\begin{aligned}
& J_{1}=2 \int \partial_{x} b_{1} \partial_{y} u_{1} j d x d y, \quad J_{2}=2 \int \partial_{x} b_{1} \partial_{x} u_{2} j d x d y, \\
& J_{3}=2 \int \partial_{x} u_{1} \partial_{y} b_{1} j d x d y, \quad J_{4}=2 \int \partial_{x} u_{1} \partial_{x} b_{2} j d x d y, \quad J_{5}=\int \partial_{x} \theta \omega d x d y .
\end{aligned}
$$

The bounds for $J_{1}, J_{2} J_{3}$ and $J_{4}$ can be found in [1], which are ( for $q$ large enough such that $q \delta>2$ )

$$
\begin{gathered}
\left|J_{1}\right| \leq \frac{1}{48}\left\|\partial_{x} \omega\right\|_{2}^{2}+\frac{\epsilon}{4}\left\|\Lambda^{\delta} j\right\|_{2}^{2}+C\left\|b_{1}\right\|_{q}^{\frac{2 q \delta}{q \delta-2}}\|j\|_{2}^{2}+\frac{1}{48}\left\|\partial_{x} j\right\|_{2}^{2} \\
+\frac{\epsilon}{4}\left\|\Lambda^{\delta} \omega\right\|_{2}^{2}+C\left\|b_{1}\right\|_{q}^{\frac{2 q \delta}{g \delta-2}}\|\omega\|_{2}^{2} \\
\left|J_{2}\right| \leq \frac{1}{48}\left\|\partial_{x} \omega\right\|_{2}^{2}+\frac{1}{48}\left\|\partial_{x} j\right\|_{2}^{2}+C\left\|\partial_{x} b_{1}\right\|_{2}^{2}\left(\|\omega\|_{2}^{2}+\|j\|_{2}^{2}\right) .
\end{gathered}
$$


We can bound $J_{3}$ similar fashion as $J_{1}$,

$$
\left|J_{3}\right| \leq \frac{1}{48}\left\|\partial_{x} \omega\right\|_{2}^{2}+\frac{1}{48}\left\|\partial_{x} j\right\|_{2}^{2}+\frac{\epsilon}{4}\left\|\Lambda^{\delta} j\right\|_{2}^{2}+C\left\|u_{1}\right\|_{q}^{\frac{2 q \delta}{q \delta-2}}\|j\|_{2}^{2} .
$$

$J_{4}$ can be bounded in a similar fashion as $J_{2}$ and

$$
\left|J_{4}\right| \leq \frac{1}{48}\left\|\partial_{x} j\right\|_{2}^{2}+C\left\|\partial_{x} u_{1}\right\|_{2}^{2}\|j\|_{2}^{2} .
$$

$J_{5}$ obeys

$$
J_{5} \leq\|\theta\|_{2}\left\|\partial_{x} \omega\right\|_{\theta} .
$$

Inserting the estimates for $J_{1}, J_{2}, J_{3}, J_{4}$ and $J_{5}$ in (4.2) yields the desired global $H^{1}$-bound for $(u, b)$. The $H^{1}$ - bound for $\theta$ is similar to the previous section. One can follow line to line from previous section to show the global $\mathrm{H}^{2}$-bound. This completes the proof of Theorem 1.3.

\section{Acknowledgements}

The authors would like to thank the referees for their valuable time, comments and suggestions to improve the presentation of this paper.

\section{References}

[1] C. Cao, D. Regmi and J. Wu, The 2D MHD equations with horizontal dissipation and horizontal magnetic diffusion, J. Differential Equations, 254 (2013), 2661-2681.

[2] C. Cao and J. Wu, Global regularity for the 2D MHD equations with mixed partial dissipation and magnetic diffusion, Adv. in Math., 226 (2011), 1803-1822.

[3] C. Cao, J. Wu and B. Yuan, The 2D incompressible magnetohydrodynamics equations with only magnetic diffusion, preprint.

[4] Q. Chen, C. Miao and Z. Zhang, The Beale-Kato-Majda criterion for the 3D magnetohydrodynamics equations, Comm. Math. Phys., 275 (2007), 861-872.

[5] Q. Chen, C. Miao and Z. Zhang, On the well-posedness of the ideal MHD equations in the Triebel-Lizorkin spaces, Arch. Ration. Mech. Anal., 195 (2010), 561-578.

[6] J. Chengn, L. Du and Z. Zhang, 2D Magnetic Bénard problem with partial viscosity, J. Math. Fluid Mech., 17 (2015), 769-797.

[7] G. Duvaut and J.-L. Lions, Inéquations en thermoélasticité et magnétohydrodynamique, Arch. Rational Mech. Anal., 46 (1972), 241-279.

[8] C. He and Z. Xin, Partial regularity of suitable weak solutions to the incompressible magnetohydrodynamic equations, J. Funct. Anal., 227 (2005), 113-152.

[9] C. He and Z. Xin, On the regularity of weak solutions to the magnetohydrodynamic equations, J. Differential Equations, 213 (2005), 235-254.

[10] Q. Jiu and D. Niu, Mathematical results related to a two-dimensional magnetohydrodynamic equations, Acta Math. Sci. Ser. B Engl. Ed,. 26 (2006), 744-756. 
[11] Q. Jiu and J. Zhao, A remark on global regularity of 2D generalized magnetohydrodynamic equations, arXiv:1306.2823v1 [math.AP] 13 Jun 2013.

[12] Z. Lei and Y. Zhou, BKM's criterion and global weak solutions for magnetohydrodynamics with zero viscosity, Discrete Contin. Dyn. Syst., 25 (2009), 575-583.

[13] F. Lin, L. Xu and P. Zhang, Global small solutions to 2-D incompressible MHD system, arXiv:1302.5877v1 [math.AP] 24 Feb 2013.

[14] F. Lin and P. Zhang, Global small solutions to MHD type system (I): 3-D case, Comm. Pure. Appl. Math., to appear.

[15] D. Regmi, A regularity criterion for two-and-half-dimensional magnetohydrodynamic equations with horizontal dissipation and horizontal magnetic diffusion, Mathematical Methods in the Applied Sciences, 40(5) (2017).

[16] D. Regmi, Global weak solutions for the two-dimensional magnetohydrodynamic equations with partial dissipation and diffusion, Nonlinear Anal.-Theor., 49(2) (2016), 157-164.

[17] D. Regmi and J. Wu, Global regularity for the 2D magneto-micropolar equations with partial dissipation, J. Math. Study, 49(2) (2016) , 169-194.

[18] T. Runst and W. Sickel, Sobolev Spaces of Fractional Order, Nemytskij Operators and Nonlinear Partial Differential Equations, Walter de Gruyter, Berlin, New York, 1996.

[19] M. Sermange and R. Temam, Some mathematical questions related to the MHD equations, Comm. Pure Appl. Math., 36 (1983), 635-664.

[20] C. Trann, X. Yu and Z. Zhai, On global regularity of 2D generalized magnetohydrodynamic equations, J. Differential Equations, 254 (2013), 4194-4216.

[21] Y. Wang, BMO and the regularity criterion for weak solutions to the magnetohydrodynamic equations, J. Math. Anal. Appl., 328 (2007), 1082-1086

[22] W. Wang and Z. Zhang, Limiting case for the regularity criterion to the 3-D magnetohydrodynamics equations, J. Differential Equations, 252 (2012), 5751-5762.

[23] J. Wu, Generalized MHD equations, J. Differential Equations, 195 (2003), 284-312.

[24] J. Wu, Regularity criteria for the generalized MHD equations, Comm. Partial Differential Equations, 33 (2008), 285-306.

[25] J. Wu, Global regularity for a class of generalized magnetohydrodynamic equations, J. Math. Fluid Mech., 13 (2011), 295-305.

[26] G. Wu and X. Zheng, Global well-posedness for the two-dimensional nonlinear boussinesq equations with vertical dissipation, preprint.

[27] B. Yuan and L. Bai, Remarks on global regularity of 2D generalized MHD equations, arXiv:1306.2190v1 [math.AP] 11 Jun 2013.

[28] K. Yamazaki, Global regularity of generalized magnetic, Bernard Problem Methods Appl. Sci., 2016 doi: 10.1002/mma.4116.

[29] K. Yamazaki, Remarks on the global regularity of two-dimensional magnetohydrodynamics system with zero dissipation, arXiv:1306.2762v1 [math.AP] 13 Jun 2013.

[30] K. Yamazaki, On the global regularity of two-dimensional generalized magnetohydrodynamics system, arXiv:1306.2842v1 [math.AP] 13 Jun 2013.

[31] Y. Zhou and J. Fan, A regularity criterion for the 2D MHD system with zero magnetic diffusivity, J. Math. Anal. Appl., 378 (2011), 169-172.

[32] Y. Zhou, J. Fan, and G. Nakamura, Global Cauchy problem for a 2D magnetic Bénard problem with zero thermal conductivity, Applied Mathematics Letters, 26 (2013), 627-530. 